\title{
Activity-Dependent Phosphorylation of Tyrosine Hydroxylase in Dopaminergic Neurons of the Rat Retina
}

\author{
Paul Witkovsky, ${ }^{1,2}$ Eleonora Veisenberger, ${ }^{1}$ John W. Haycock, ${ }^{4}$ Abram Akopian, ${ }^{1}$ Antonio Garcia-Espana, ${ }^{3}$ and \\ Emanuel Meller ${ }^{3}$ \\ Departments of ${ }^{1}$ Ophthalmology, ${ }^{2}$ Physiology and Neuroscience, and ${ }^{3}$ Psychiatry, New York University School of Medicine, New York, New York 10016, \\ and ${ }^{4}$ Department of Biochemistry and Molecular Biology, Louisiana State University, Baton Rouge, Louisiana 70803
}

We studied in vivo activity-dependent phosphorylation of tyrosine hydroxylase (TH) in dopaminergic (DA) neurons of the rat retina. TH phosphorylation (TH-P) was evaluated by immunocytochemistry, using antibodies specific for each of three regulated phosphorylation sites. TH synthesis rate was measured by dihydroxyphenylalanine (DOPA) accumulation in the presence of NSD-1015, an inhibitor of aromatic amino acid decarboxylase. TH-P was increased markedly by light or after intraocular injection of $\mathrm{GABA}_{\mathrm{A}}$ and glycine inhibitors. All three phosphospecific antibodies responded similarly to test drugs or light. A 30 min exposure to light increased DOPA accumulation by threefold over that seen after $30 \mathrm{~min}$ in darkness. Immunostaining to an anti-panNa channel antibody was found in all parts of the DA neuron. TTX blocked TH-P induced by light or GABA/glycine inhibitors but only in varicosities of the DA axon plexus, not in perikarya or dendrites. Veratridine increased TH-P in all parts of the DA neuron. The distribution of the monoamine vesicular transporter 2 was shown by immunocytochemistry to reside in varicosities of the DA plexus but not in dendrites, indicating that the varicosities are sites of dopamine release. Collectively, these data indicate that, in the retina, dopamine synthesis in varicosities is affected by the spiking activity of retinal neurons, possibly including that of the DA neurons themselves.

Key words: retina; dopaminergic neuron; tyrosine hydroxylase; phosphorylation; TTX; monoamine vesicular transporter 2

\section{Introduction}

Dopamine contributes importantly to day/night-induced alterations of retinal signal transmission (for review, see Witkovsky, 2004). It is released by a unique set of retinal amacrine or interplexiform cells, from which dopamine diffuses to target neurons and non-neuronal cells distributed throughout the retina. All major types of retinal cells have receptors for dopamine (Nguyen-Legros et al., 1999). In a reciprocal way, the dopaminergic (DA) neurons are the recipients of light-initiated signals that influence dopamine production and release. The importance of an extrinsic regulation of dopaminergic neurons is evidenced by the finding that, in retinas of mice with absent or defective photoreceptor outer segments, dopamine synthesis and use are markedly reduced compared with wild-type mice (Nir et al., 2000a,b).

The regulation of dopamine production and release in the retina is beginning to be understood. Gustincich et al. (1997) showed that retinal dopaminergic neurons have an intrinsic spiking rate that is modulated up or down by excitatory and inhibi-

Received Dec. 10, 2003; revised March 17, 2004; accepted March 24, 2004.

This work was supported by National Institutes of Health Grants EY 03570 (P.W.), EY12497 (A.A.), NS 25134 (J.W.H.), and MH 60592 (E.M.).

Correspondence should be addressed to Dr. Paul Witkovsky, Department of Ophthalmology, New York University School of Medicine, 550 First Avenue, New York, NY 10016. E-mail: pw20@nyu.edu.

A. Garcia-Espana's present address: Research Unit, University Hospital Joan XXIII, Mallfre Guash 4, 43007 Tarragona, Spain.

D01:10.1523/JNEUROSCI.5436-03.2004

Copyright $\odot 2004$ Society for Neuroscience $\quad$ 0270-6474/04/244242-08\$15.00/0 tory amino acid neurotransmitters. Puopolo et al. (2001) demonstrated that dopamine release by these neurons is both a calcium-dependent process and blocked by tetrodotoxin.

It has long been appreciated that production and release of catecholamines are closely linked (for review, see Zigmond et al., 1989), suggesting that the mechanisms that modify the spiking rate might also influence dopamine synthesis; the present investigation examined this possibility in a mammalian retina. In a previous study (Witkovsky et al., 2000), we used three antibodies specific for each of the three regulated phosphorylation sites on tyrosine hydroxylase (TH), the rate-limiting enzyme for dopamine synthesis. We found that light, or certain neurochemicals, induced strong TH phosphorylation (TH-P). Phosphorylation confers on $\mathrm{TH}$ a greater affinity for its tetrahydrobiopterin cofactor, resulting in an increased rate of dopamine synthesis (Kumer and Vrana, 1996). Each TH phosphorylation site is controlled by a different, but partially overlapping, group of kinases (Haycock and Haycock, 1991) (for review, see Kumer and Vrana, 1996). In the present study, we test the effects of modifying spiking activity on TH phosphorylation. We observed that the action of the neurotoxin TTX was selective, eliminating TH phosphorylation in varicosities of the dopaminergic cell axon plexus but not in dopaminergic cell bodies or dendrites. On the other hand, veratridine, which increases the spiking rate, augmented TH phosphorylation in all parts of the DA neuron. These findings emphasize that, for DA neurons in both retinal and nigrostriatal systems (Grace and Bunney, 1983), spiking activity is an important regulatory component of dopamine synthesis and release. 


\section{Materials and Methods}

Animals and animal surgery. Long-Evans rats were obtained from a commercial supplier (Taconic, Germantown NY) and maintained in the animal care facility on a $12 \mathrm{hr}$ light/dark cycle with access to food and water ad libitum. The day before the experiment, the rats were transferred to a light-tight chamber at 5:00 P.M. Experiments began on the following day between 8:00 and 9:00 A.M. All anesthetic and intraocular injection procedures were performed in complete darkness, with the aid of an infrared viewer ("Nitemare"; B. E. Meyers \& Co., Redmond, WA). The animal was first exposed to $100 \% \mathrm{CO}_{2}$ for $30 \mathrm{sec}$ and then injected with $110 \mathrm{mg} / \mathrm{kg}$ ketamine and $11 \mathrm{mg} / \mathrm{kg}$ xylazine intraperitoneally. After anesthesia had occurred (no withdrawal response to strong leg pinch), the rat was placed on a holder and observed with an infrared television camera. The animal handling and anesthetic procedures we used conform to National Institutes of Health guidelines and were approved by the Animal Care Committee of New York University School of Medicine.

Two drops of lidocaine were administered to the corneal surface, and $10 \mu \mathrm{l}$ of Ringer's solution containing the test drug was injected into the left eye, using a pipetter modified to accept a 27 gauge needle. The right eye received an injection of drug-free Ringer's solution. To ensure accurate injection volumes, it was necessary to backfill the needle with the test solution before connecting it to the pipetter. The eye volume of the $175-200$ gm body weight rats we used is $\sim 50 \mu \mathrm{l}$, so drug concentrations injected were five times the target dosage. Sham injections, injections of $10 \mu \mathrm{l}$ of drug-free Ringer's solution, or injections of $10 \mu \mathrm{l}$ of Ringer's solution containing $0.1 \mu \mathrm{l}$ of ethyl alcohol (used to dissolve veratridine; see below) did not alter TH-P or panTH immunoreactivity (IR).

For experiments requiring darkness, after an additional 20-30 min, the anesthetized rat was decapitated in darkness, the eyes were enucleated, and the cornea was cut away under infrared illumination. The eyecup was placed in freshly prepared, $4 \%$ paraformaldehyde in $0.1 \mathrm{M}$ phosphate buffer, $\mathrm{pH}$ 7.2. After $5 \mathrm{~min}$, the tissues were transferred to light, and the retina was dissected free and fixed another $55 \mathrm{~min}$.

Sample sizes. For the various experiments, a ratio is given indicating the fraction of trials in which the experimental retina differed clearly from the control retina.

Immunocytochemistry. After fixation, the retina was cut into small squares, 2-3 $\mathrm{mm}$ on a side. These pieces were washed three times for $20 \mathrm{~min}$ each in PBS and then left for $1 \mathrm{hr}$ in blocking solution (PBS containing 10 $\mathrm{mg} / \mathrm{ml}$ bovine serum albumin, $0.3 \%$ Triton $\mathrm{X}-100$, and $0.1 \% \mathrm{Na}$ azide). Thereafter, the pieces were incubated $16-20 \mathrm{hr}$ in the primary antibody diluted in blocking solution. The primary antibodies were as follows: mouse monoclonal anti-TH (1:500; Chemicon, Temecula, CA); rabbit antityrosine hydroxylase (1:500; Chemicon), anti-serine 19-phosphotyrosine hydroxylase (THP19) (1:25,000), anti-serine 31-phosphotyrosine hydroxylase (THP31) (1:12,500), anti-serine 40-phosphotyrosine hydroxylase (THP40) (1:5000), mouse monoclonal anti-sodium channel (pan) (1:500; Sigma, St. Louis, MO), and anti-vesicular monoamine transporter 2 (VMAT2) (1:3000). The three phosphospecific antibodies were produced by J. W. Haycock and have been shown in Western blots to stain only a single band in rat brain preparations (Salvatore et al., 2000). The anti-VMAT2 antibody also was produced by J. Haycock (Haycock et al., 2003) using a method described by Erickson et al. (1996).

After washes in PBS (three times for 20 min each), the tissues were placed for $2 \mathrm{hr}$ in a mixture of Cy3-conjugated donkey anti-rabbit (1:200; Jackson ImmunoResearch, West Grove, PA) and Alexa 488-conjugated goat anti-mouse (1:400; Molecular Probes, Eugene, OR) secondary antibodies diluted with blocking solution. After a final series of washes in PBS (three times for $20 \mathrm{~min}$ each), the pieces were mounted flat, vitreous side up, and coverslipped with VectaShield (Vector Laboratories, Burlingame, CA). As controls, we omitted the primary antibodies and saw no staining of cell bodies or processes. Additionally, we note that immunostaining with phosphospecific anti-TH or anti-VMAT2 antibodies invariably colocalized with anti-panTH immunostaining and was found nowhere else. Because TH-P immunostaining intensity was the main experimental variable, only retinal pieces in which anti-panTH immunostaining was robust and homogeneous were used for analysis.

For one set of experiments in which two antibodies made in rabbit were applied to the same section, we used the Zenon IgG labeling kit of Molecular Probes, with Alexa fluors 488 and 555. Anti-phosphotyrosine hydroxylase antibodies were separately incubated with an Alexa fluor for $5 \mathrm{~min}$ in the ratio of 1:6, followed by the blocking solution in the same ratio and then applied to $14 \mu \mathrm{m}$ cryostat sections of retina for $2 \mathrm{hr}$. Higher concentrations of primary antibody than those used in conventional immunocytochemistry were required: THP19 at 1:1000, THP31 at 1:500, and THP40 at 1:200. After three washes for 10 min each in PBS, the sections were fixed $5 \mathrm{~min}$ in $4 \%$ buffered paraformaldehyde, according to the directions of the manufacturer.

The retinas were viewed in a Nikon (Tokyo, Japan) Eclipse PM 800 confocal microscope equipped with a digital camera controlled by the Spot software program. Digital files were processed in Adobe Photoshop 5.5 and Adobe Illustrator 9.0 (Adobe Systems, San Jose, CA).

Whole-cell recording. Eyes were injected with either TTX or drug-free Ringer's solution using the anesthetic and surgical procedures described above. After enucleation, the retina was removed and placed receptor side up on a Millipore (Bedford, MA) filter, and $150 \mu \mathrm{m}$ slices were prepared. Whole-cell voltage-clamp recordings were obtained in a conventional way using an Axopatch 200 amplifier (Axon Instruments, Foster City, CA). Electrode resistance was typically 5-8 $\mathrm{M} \Omega$ in the bath solution. Series resistance was compensated $(70-80 \%)$ by a standard circuit. Currents were filtered at $1 \mathrm{kHz}$ by a low-pass Bessel filter on the Axopatch amplifier. The patch pipette was filled with a standard solution containing the following (in mM): $100 \mathrm{CsCl}, 2 \mathrm{MgCl}_{2}, 0.2 \mathrm{CaCl}_{2}, 2$ EGTA, 10 HEPES, 2 ATP, and 0.1 GTP, adjusted to $\mathrm{pH} 7.3$ with $\mathrm{CsOH}$ (for additional details of the method, see Akopian and Witkovsky, 2001). Neurons in the ganglion cell layer were selected for study. A standard set of depolarizing steps in $20 \mathrm{mV}$ increments from a holding potential of $-70 \mathrm{mV}$ was applied.

HPLC for electrochemical detection of dihydroxyphenylalanine. Frozen retinas were homogenized by sonication in $200 \mu \mathrm{l}$ of $0.2 \mathrm{~N}$ perchloric acid containing $1 \mathrm{ng}$ of dihydroxybenzylamine as internal standard. After centrifugation $(5 \mathrm{~min}, 15,000 \times \mathrm{g}), 150 \mu \mathrm{l}$ of supernatant was transferred to tubes containing $10 \mathrm{mg}$ of activated alumina and $600 \mu \mathrm{l}$ of adsorption buffer ( $1 \mathrm{~m}$ Tris, $\mathrm{pH} 8.6$, containing $0.6 \mathrm{~mm} \mathrm{NaHSO}_{3}$ and $0.1 \mu \mathrm{M}$ EDTA). After extraction of catechols by shaking for $20 \mathrm{~min}$, tubes were centrifuged briefly, the supernatant was discarded, and the alumina was washed twice with $0.5 \mathrm{ml}$ of wash buffer (10 mM Tris, $\mathrm{pH}$ 8.6, containing $7 \mu \mathrm{M} \mathrm{NaHSO}$ ). Catechols were eluted by shaking for 20 min with $100 \mu \mathrm{l}$ of $0.2 \mathrm{~N}$ perchloric acid, and $20 \mu \mathrm{l}$ of the resulting supernatant was analyzed for dihydroxyphenylalanine (DOPA) by HPLC using ESA (Chelmsford, MA) instrumentation: an HR-80 column and a model $5100 \mathrm{~A}$ coulometric detector equipped with a model 5011 dual-electrode high-sensitivity analytical cell. The working electrode potentials were +0.05 and $-0.25 \mathrm{~V}$; the mobile phase was ESA CAT-A-PHASE supplemented with $5 \%$ methanol at a flow rate of $1 \mathrm{ml} / \mathrm{min}$. DOPA was quantitated by reference to the internal standard.

Chemicals. TTX citrate, veratridine, strychnine, and bicuculline methiodide were obtained from Sigma. The $\mathrm{GABA}_{\mathrm{A}}$ antagonist SR95331 was obtained from Research Biochemicals (Natick, MA). Veratridine was prepared as a $10 \mathrm{~mm}$ stock in ethanol, and TTX citrate was prepared as a $3 \mathrm{~mm}$ stock in distilled water. Other drugs were dissolved directly in the Ringer's solution.

\section{Results}

\section{Light-dark differences in TH phosphorylation}

Although light-induced TH-P was established in a previous study in which eyecups were studied in vitro (Witkovsky et al., 2000), it was repeated here under in vivo conditions (five of five). For the intact rat, we found that it is crucial to exclude exposure even to very dim lights during the preparatory procedures (see Materials and Methods). Otherwise, even under nominally dark conditions, substantial TH-P is observed with all three antiphosphoTH antibodies. In complete darkness, however, TH-P immunostaining was typically weak to absent for THP31 and THP40 and present at moderate levels for THP19 (Fig. 1d-f). When TH-P is weak, it is confined to the DA perikaryon and the 
initial portions of the primary dendrites (Fig. 1f). Light or darkness do not affect anti-panTH immunoreactivity (Fig. 1, compare $a-c$ with $g-i$ ). One member of a second class of small amacrine cell showing $\mathrm{TH}$ immunoreactivity (Mariani and Hokoc, 1988) is indicated by an arrow in Figure $1 g$ but is not considered further in this report. Exposure to fluorescent room light for 20-30 $\mathrm{min}\left(\sim 30 \mu \mathrm{W} / \mathrm{cm}^{-2}\right.$ at table surface) induced substantial TH-P (Fig. $1 j-l$ ), a result obtained whether or not the rat was anesthetized during exposure to light. When TH-P is strong, it is evident throughout the cell, including the cell body, principal dendrites, and the fine processes of the DA axon plexus. When observed at higher magnification (Fig. 2a), the plexus consists of a mixture of fine $(<0.2 \mu \mathrm{m})$, straight processes, which occasionally manifest varicosities, typically $0.5-0.8 \mu \mathrm{m}$ in diameter, as well as rings $\sim 7-8 \mu \mathrm{m}$ in diameter, in which four to eight varicosities are connected by fine processes of constant diameter. When comparing identical fields of the DA plexus immunostained with anti-panTH and an antiphosphoTH antibody (Fig. 2a), we observed that the colocalization of the two antibodies was extensive but not complete, even under conditions that induced strong TH-P, e.g., exposure to room light. That is, invariably, a fraction of the fine $\mathrm{TH}$ processes and varicosities lacked phosphorylated $\mathrm{TH}$ or, to be more precise, lacked immunoreactivity for the particular anti-phosphoTH antibody being tested. In contrast, larger dendrites (Fig. $2 a$, arrow) always exhibited strong TH-P in light-exposed retinas and appear yellow in the merged image.

The finding that some varicosities lacked phosphorylated $\mathrm{TH}$ when neighboring varicosities manifested it suggested the possibility that adjacent varicosities might be immunoreactive to different anti-phosphoTH antibodies. To explore this possibility, we applied two anti-phosphoTH antibodies to the same section, using the Zenon antibody conjugates directed against rabbit IgG (see Materials and Methods). Pairs of THP19-THP31 or THP19THP40 were examined. In almost every case, the fine DA terminals were reactive to both tested antibodies (Fig. $2 b$ ), indicating that there was not a segregation of phosphorylation pathways in adjacent varicosities (a rare exception is illustrated by an arrow in Fig. 2b). When comparing Figure 2, $a$ and $b$, it is important to note that, in the latter, there is no panTH immunostaining, so the numerous processes in Figure $2 a$ that lack any TH-P are not visible in Figure $2 b$.

\section{Light increases DOPA synthesis}

To test whether TH-P was associated with an increase in dopamine production, we administered the aromatic acid decarboxylase inhibitor NSD-1015 (100 mg/kg) subcutaneously to darkadapted rats. Thereafter, the rat was either left in darkness for 30
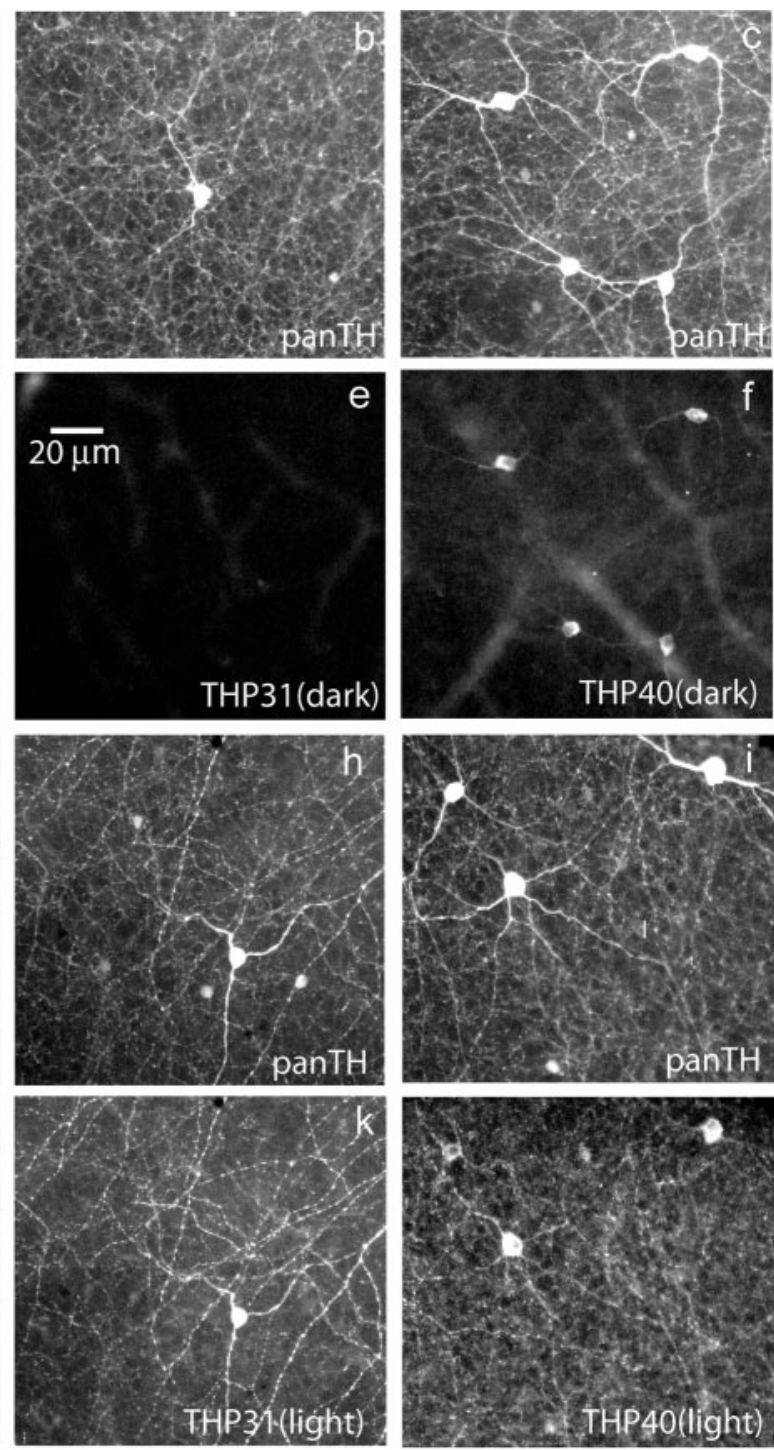

Figure 1. Influence of light and dark on TH-P. Vertical pairs ( $a / d, b / e$, etc.) show identical fields immunostained with panTH or one of three phosphospecific TH antibodies, as labeled. The top two rows are from a retina removed from a fully dark-adapted rat, immunostaining, whereas light greatly increases phosphospecific TH(THP19, THP31, and THP40) immunoreactivity, particularly in the DA plexus of fine processes and rings. The dim background staining in e and $f$ are blood vessels. Scale bar in e applies to all panels.

min or exposed to room lights for $30 \mathrm{~min}$, after which the retinas were processed for DOPA content. At time 0, before the NSD-1015 injection, retinas contained, on average, $0.54 \pm 0.07 \mathrm{ng}$ of DOPA per milligram of protein. In the presence of NSD-1015, after $30 \mathrm{~min}$ in darkness, DOPA rose to a mean of $1.79 \pm 0.28 \mathrm{ng} / \mathrm{mg}$ protein, whereas after $30 \mathrm{~min}$ light, the levels were $4.34 \pm 0.57 \mathrm{ng} / \mathrm{mg}$ protein. Thus, exposure to light increased DOPA synthesis by a factor of 3.04 over that seen in darkness $(p<0.05 ; n=4)$.

\section{Identification of $\mathrm{Na}$ channels in retinal DA neurons}

Gustincich et al. (1997) reported that mouse retinal DA neurons in culture produced TTX-sensitive action potentials. Because the DA cells they studied lacked most processes, we investigated the distribution of voltage-gated $\mathrm{Na}$ channels in DA neurons. The antibody we used (see Materials and Methods) is reported to stain optic axons in the rat retina (Boiko et al., 2003). Figure 3 illustrates vertical sections of rat retina coimmunostained with antipanNa (Fig. 3a) and anti-TH (Fig. 3b). The anti-panNa antibody 

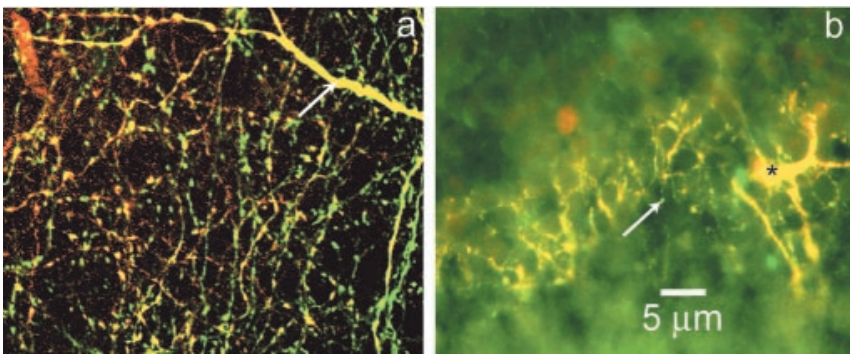

Figure 2. Extent of phosphospecific TH-IR in the DA plexus. a, Double immunostaining with anti-THP19 (red) and anti-panTH (green). Colocalization is represented by yellow. The DA plexus consists of fine processes containing multiple varicosities. A portion of a DA dendrite is indicated by an arrow. Note that not all elements of the plexus are immunoreactive for the THP19 antibody. b, Colocalization of anti-THP19 (red) and anti-THP40 (green) in the DA plexus, using the Zenon technique (see Materials and Methods). Almost all of the varicosities (seen here as small, variously shaped profiles) colocalize the two phosphospecific antibodies. A rare exception is illustrated by the arrow. ${ }^{*}$ indicates a portion of a DA dendrite. Scale bar applies to both panels.
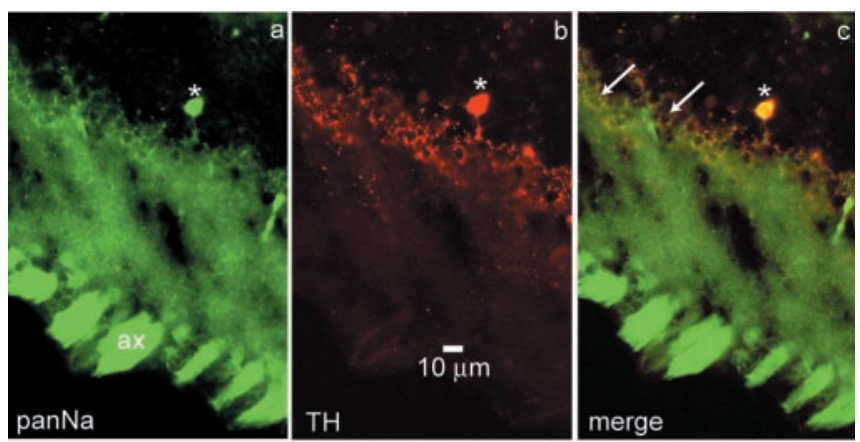

Figure 3. Colocalization of $\mathrm{TH}$ and panNa-IR in DA neurons. $a$, Vertical section of retina immunostained with an anti-panNa antibody. Note panNa-IR in DA cell body (asterisks) and rings (arrows) in outer portion of the IPL and ganglion cell axons (ax). $b$, The identical field immunostained for TH-IR. * indicates DA perikaryon. The DA plexus of rings also is visible. $c$, Merged image showing colocalization of TH- and panNa-IR in DA cell body $\left(^{*}\right)$ and DA plexus. Arrows indicate colocalization in the corresponding three axon terminal rings of $a$.

labeled both optic nerve axons (ax) and also DA neurons; both the perikaryon and the axonal rings of the DA neurons were immunoreactive. In the merged image (Fig. $3 c$ ), these components of DA neurons are yellow, indicating colocalization of TH and voltage-gated Na channels.

\section{Intraocular injection of TTX is effective in blocking spiking Na currents}

As a preliminary to testing the effects of TTX on TH-P induction, we wanted to verify that the injected TTX blocked retinal spiking activity. Accordingly, a $10 \mu \mathrm{l}$ injection of Ringer's solution containing $15 \mu \mathrm{M}$ TTX was administered to one eye of the rat, whereas the control eye was injected with $10 \mu$ l of Ringer's solution. Thereafter, each retina was prepared for whole-cell patch recording. Because DA neurons are not identifiable in unstained slices, we surveyed neurons in the ganglion cell layer. The current-voltage profiles of neurons from the Ringer's solutioninjected eye had fast inward currents characteristic of spiking $\mathrm{Na}$ currents (eight of eight) (Fig. 4, left), whereas in the eye receiving TTX, the fast inward current was blocked (six of six) (Fig. 4, right). Outward currents were unaffected by TTX.

\section{TH-P induced by neurochemicals}

Although light is a potent stimulator of TH-P, its disadvantage is that it activates multiple retinal pathways, using a large number

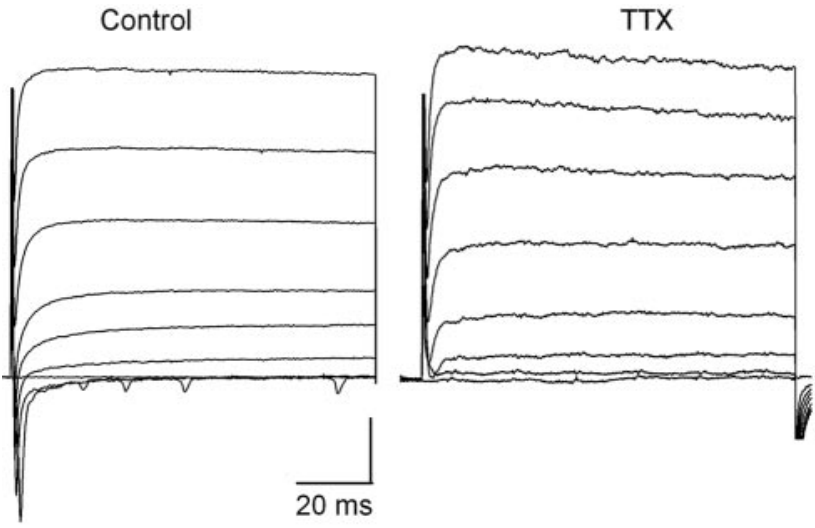

Figure 4. Voltage-clamp profiles of rat retinal spiking neurons. Whole-cell patch-clamp records were obtained from neurons in the ganglion cell layer in rat retinal slices. Neurons were held at $-70 \mathrm{mV}$ and then stepped in $20 \mathrm{mV}$ depolarizing increments to $+70 \mathrm{mV}$. Left, In slices from control retina $(n=8)$, the voltage-clamp profile consists primarily of a rapid inward current and a slower, sustained outward current. Right, In retinal slices from a TTX-treated eye $(n=6)$, the fast inward current is selectively blocked

of different neurotransmitters and neuromodulators. Therefore, we tested various neurochemicals injected directly into the eye (compare Materials and Methods) to find one or more that induced TH-P and with a mode of action that would be easier to interpret. Two categories of neurochemical proved useful in this regard: $\mathrm{GABA}_{\mathrm{A}}$ blockers and veratridine, which increases spike firing (see below). GABA blockers were tested because the DA neurons possess GABA receptors of this subtype (Feigenspan et al., 2000). Bicuculline (100 $\mu \mathrm{M})$ or SR95331 (20 $\mu \mathrm{M})$ were equally effective (four of five). The glycine receptor antagonist strychnine was added together with the $\mathrm{GABA}_{\mathrm{A}}$ blocker, because DA neurons also are inhibited by glycine (Gustincich et al., 1997).

In the experiment illustrated in Figure 5, a mixture of bicuculline $(100 \mu \mathrm{M})$ and the glycine blocker strychnine $(20 \mu \mathrm{M})$ was injected into one eye; the other eye received the same bicucullinestrychnine mixture together with TTX $(15 \mu \mathrm{M})$ (six of nine). After $30 \mathrm{~min}$ in darkness, the rat was killed, and the retinas were processed for immunocytochemistry. In the absence of TTX, the $\mathrm{GABA}_{\mathrm{A}}$ and glycine blockers induced strong TH-P (Fig. 5b,f,j). These data illustrate that the same result was achieved with all three phosphospecific TH antibodies. In the TTX-treated eye, TH-P was evident in DA perikarya and primary dendrites, but it was absent in the plexus of dopaminergic fibers (Fig. $5 d, h, l$ ). An identical result was obtained when TTX alone was injected into one eye and the rat was then exposed to room light for $30 \mathrm{~min}$ before being killed (four of four; data not illustrated). On the other hand, comparison of control and TTX-treated retinas immunostained with anti-panTH (Fig. 5a,c,e,g) shows that TTX did not affect the distribution or staining intensity obtained with the anti-panTH antibody in the DA axon plexus.

\section{Distribution of VMAT2 in dopaminergic neurons}

The results obtained with TTX indicate that blockage of retinal spiking activity selectively inhibits TH-P in DA terminals. Dacey (1990) suggested that the rings are, in fact, the axon terminals. Electron microscopy (Kolb et al., 1990) showed that varicosities found in axons and in rings are sites of agranular vesicle accumulation and associated presynaptic specializations. We therefore looked at the distribution of the vesicular monoamine transporter 2, which is found in close association with the catecholamine release sites in the brain (Hoffman et al., 1998). 
Figure $6 a-c$ shows that VMAT2 is associated selectively with the DA axon plexus. Figure $6 a$ illustrates immunostaining with anti-VMAT2 alone, documenting that VMAT2 is contained within varicosities, typically arrayed in rings. In addition, an irregular patch of VMAT2 (Fig. $6 a$, oval) is found within DA perikarya, as verified in Figure $6 b$, in which the VMAT2 profile is merged with the same retinal area coimmunostained with an anti-panTH antibody. In the merged image, it is evident that DA dendrites lack VMAT2, whereas DA cell bodies have a patch of VMAT2 not associated with the cell membrane. Puopolo et al. (2001) concluded that this patch is associated with the Golgi apparatus, possibly reflecting newly synthesized VMAT2. One ring double immunostained with anti-VMAT2 and anti-panTH is indicated by correspondingly positioned arrows in Figure 6, $a$ and $b$.

Although the great majority of overlap of VMAT2 and TH immunostaining occurs in the rings, within the main dopaminergic plexus, VMAT2 also is associated with varicosities occurring in the straight portions of individual axons (Fig. $6 c$, top arrow). Presumably, these represent sites of en passant synapses made by axons. Just as was found for TH-P (compare with Fig. 2 ), not every ring or every varicosity in the primary plexus contains VMAT2 (Fig. $6 b, c)$. Exposure of the retina to TTX had no effect on VMAT2 immunoreactivity (data not illustrated).

In addition to the main DA plexus found in the most distal sublamina (sublamina 1) of the inner plexiform layer (IPL), additional $\mathrm{TH}$-immunoreactive processes extend distally toward the layer of photoreceptors (Savy et al., 1995) and also penetrate more deeply into the IPL (Kolb et al., 1990). We examined whether these processes also manifested antiVMAT2 immunostaining. Figure $6, d$ and $e$, illustrates distal processes immunostained with both anti-panTH and antiVMAT2. Such processes are characterized by straight segments connecting infrequent varicosities. As was the case for the main DA plexus, some varicosities exhibited anti-VMAT2 immunostaining (Fig. $6 d$, arrows), whereas others lacked it (Fig. $6 d$, circles at top left). Similarly, the THimmunoreactive processes in the middle of the IPL showed infrequent anti-VMAT2positive varicosities (Fig. 6e).
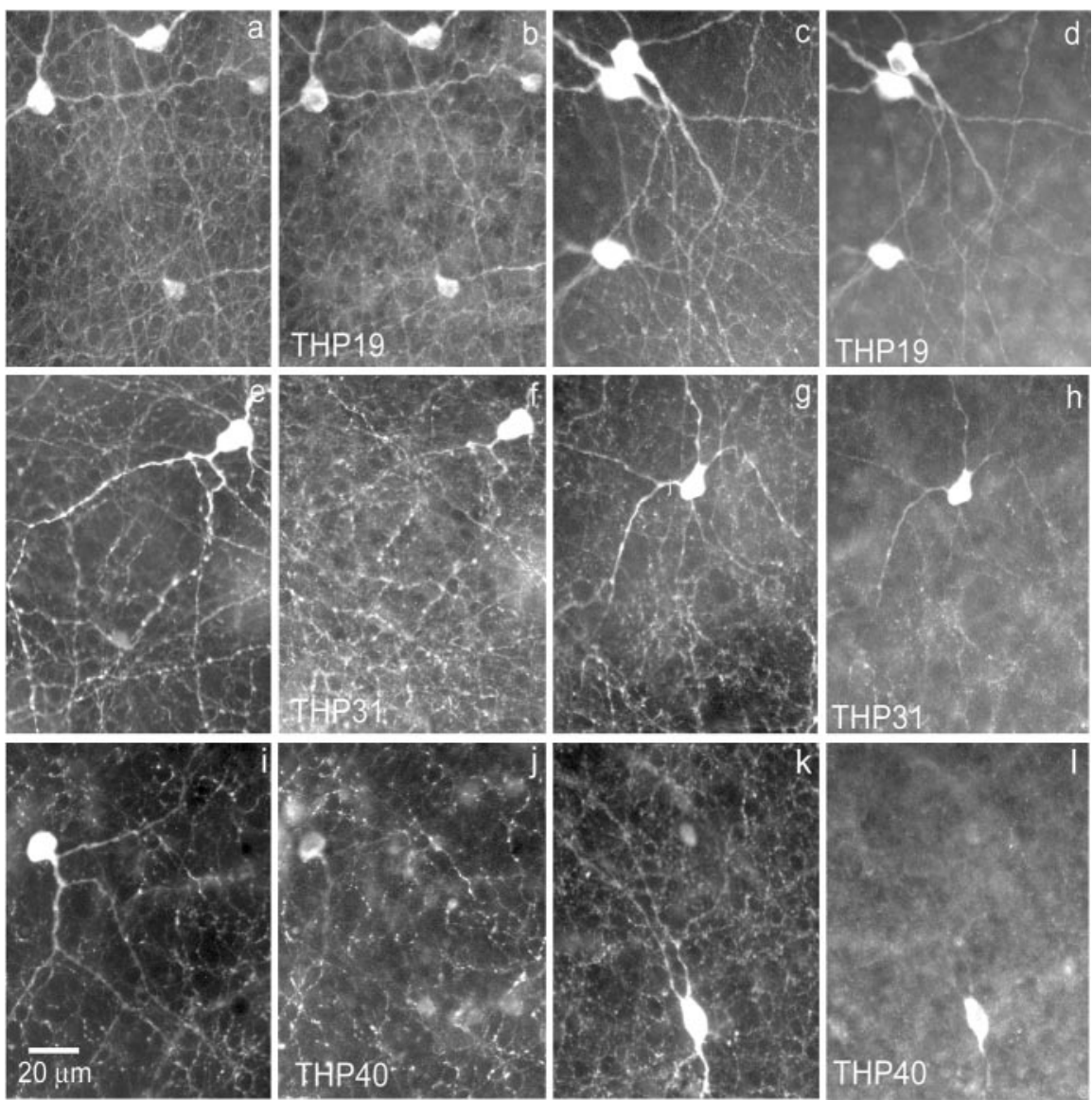

Gaba/gly blockers
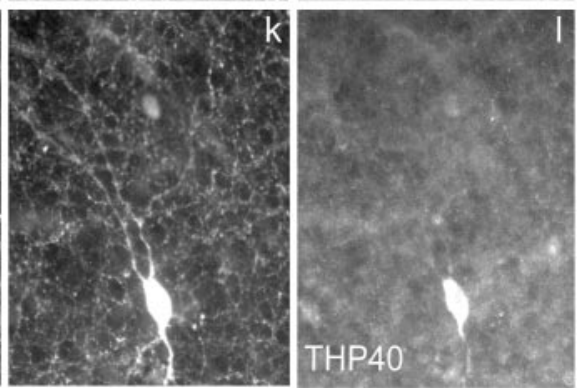

Gaba/gly blockers + TTX

Figure 5. Effect of TTX on TH-P immunoreactivity. Left-right pairs (a/b, etc.) show identical fields immunostained with anti-panTH (left member of the pair) and one of three anti-phosphoTH antibodies, as labeled. $a / b, e / f, i / j$, Left eye injected in darkness with bicuculline $(100 \mu \mathrm{m})$ and strychnine $(20 \mu \mathrm{M}) . c / d, g / h, k / l$, Right eye of same rat injected in darkness with bicuculline, strychnine, and TTX (15 $\mu \mathrm{m})$. Bicuculline plus strychnine alone induced THP in DA cell bodies, dendrites, and DA plexus $(b, f, j)$. In the presence of TTX, bicuculline, plus strychnine, THP is weak or absent in the DA plexus $(d, h, l)$ but is present in DA cell bodies and dendrites. TTX did not affect anti-panTH-IR (compare $a, e, i$ with $c, g, k)$. Scale bar in $i$ applies to all panels. gly, Glycine.
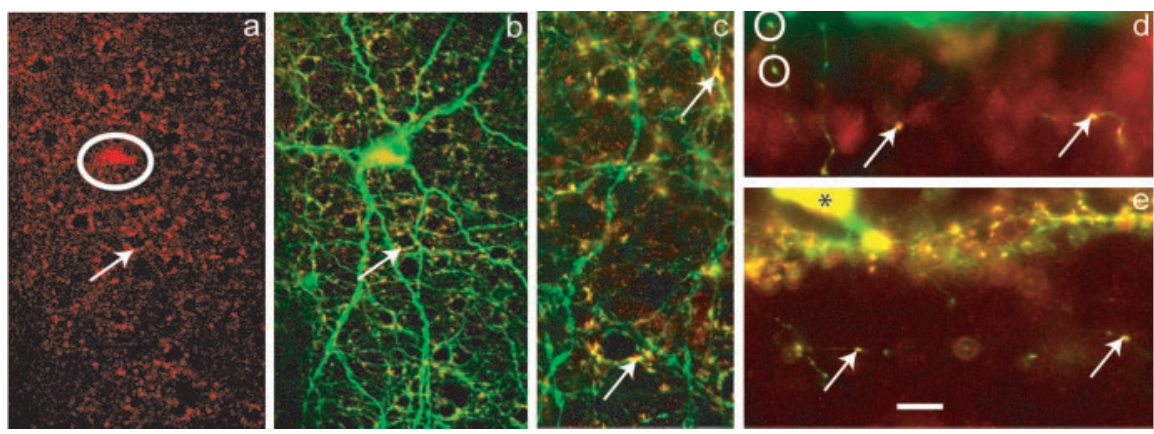

Figure 6. VMAT2 immunoreactivity in rat retinal DA neurons. Anti-VMAT2-IR alone ( $a$ ) and combined anti-VMAT2 and antipanTH-IR $(b)$. Note the patch of anti-VMAT2-IR in cell body (oval in a) but otherwise mainly in rings. Two representative corresponding rings in $a$ and $b$ are indicated by arrows. $c$, Colocalization of anti-VMAT2- and anti-panTH-IR in the DA plexus. Top arrow indicates colocalization in an axonal varicosity, and bottom arrow indicates colocalization in a ring. Note that not all varicosities show anti-VMAT2-IR. $d$, Arrows indicate colocalization of anti-VMAT2-IR and anti-panTH-IR in varicosities within the INL. Circles surround two varicosities that lack anti-VMAT2-IR. e, Similar colocalization of anti-VMAT2-IR and anti-panTH-IR in varicosities found in the middle of the IPL (arrows). The contrast of this photo was increased to make the small varicosities more visible, resulting in an overexposure of the DA cell body (asterisks). Scale bars (in $d$ ): $a, b, 10 \mu \mathrm{m} ;(-e, 5 \mu \mathrm{m}$.

\section{Effect of veratridine on TH-P}

Veratridine is a sodium channel agonist that increases the spiking rate (Hille, 1992). A single injection of veratridine $(20 \mu \mathrm{M})$ into one eye (four of four) increased TH-P modestly over dark control levels (data not illustrated), but a more robust and consistent result was obtained with veratridine at $100 \mu \mathrm{M}$ (three of three). A comparison of $b$ and $d$ in Figure 7 reveals that exposure to veratridine $(d)$ substantially increases TH-P at serine 19 compared with that seen in a 

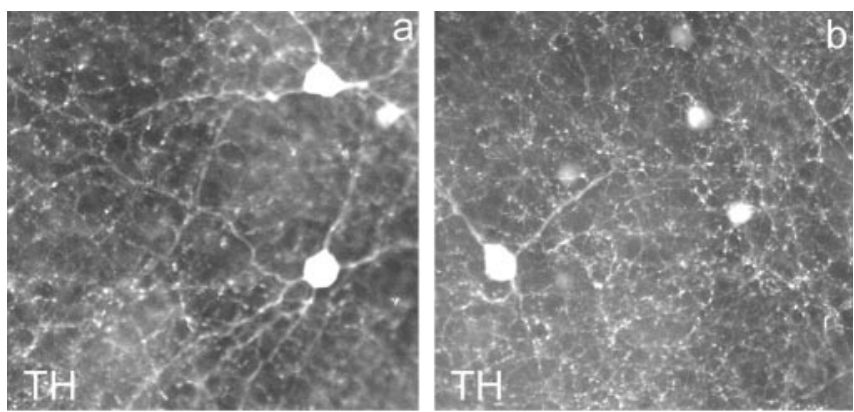

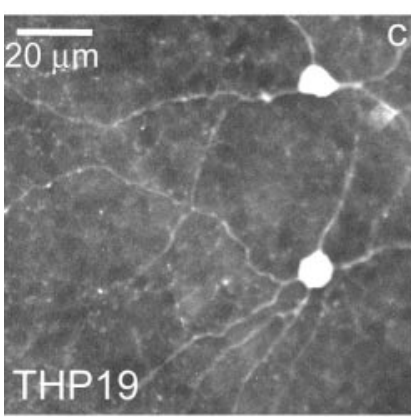

control

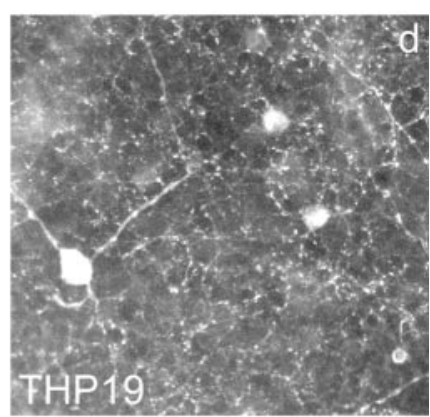

veratridine
Figure 7. Effects of veratridine on TH-P immunoreactivity. Horizontal pairs $(a / b, c / d)$ illustrate identical fields immunostained for panTH (left) or anti-serine 19 phosphotyrosine hydroxylase (right). $a / b$, Eye received sham injection in darkness. Note absence of THP19 in DA plexus (b). $c / d$, Left eye injected with veratridine $(100 \mu \mathrm{m})$ in darkness. Veratridine induces strong THP19 in the DA plexus (d). Scale bar in $d$ is for all panels.

dark control $(b)$. Similar veratridine-induced increases in TH-P were observed at serines 31 and 40 (data not illustrated). On the other hand, exposure to veratridine did not affect panTH immunoreactivity [Fig. 7, compare $a$ (control), $c$ (veratridine-treated)].

\section{Discussion}

Two principal findings emerged from this study. The first is that the main sites of dopamine release, as defined by the immunocytochemical localization of VMAT2, are varicosities located in the DA axon plexus. Most VMAT2-containing varicosities are associated with ring structures, an arrangement of four to seven varicosities separated by fine tube-like segments, the whole ring being $\sim 7 \mu \mathrm{m}$ in diameter (Fig. 6, compare $a, b$ ). Other varicosities were seen to be local swellings of fine axon-like processes. Additional VMAT2-positive varicosities were very sparsely distributed in the inner nuclear layer (INL) and the middle portions of the inner plexiform layer. The distally directed processes emerge from the large DA neurons (Savy et al., 1995), and it is these neurons that are the main focus of the present study. Some or all of the processes in the central portion of the inner plexiform layer may derive from the small, type II TH-immunoreactive neurons (Mariani and Hokoc, 1988). In contrast, the perikarya and dendrites of the DA cell lack VMAT2, except for a patch in the perikaryon that Puopolo et al. (2001) characterized as being associated with the Golgi apparatus. This patch presumably represents newly synthesized VMAT2 in the process of being transported to the axon terminals.

DA neurons are unusual in having TH distributed throughout the neuron, including the perikaryon, dendrites, axons, and axon terminals. We noted that activity-dependent phosphorylation of $\mathrm{TH}$ extended throughout the main processes of the DA neuron but was present in most but not all of the varicosities (compare Fig. 2a). We reject the idea that varicosities not exhibiting TH-P emanate from the small, type II TH neurons (Mariani and Hokoc, 1988) on the following grounds: (1) type II TH neurons do not arborize in the distalmost sublamina of the IPL, which is the focal plane of Figure $2 a$; (2) type II neurons show very weak TH-IR, whereas the processes we examined had robust TH-IR; and (3) some processes (compare Fig. 2a) show a mixture of phosphorylated and nonphosphorylated varicosities. An alternative explanation based on the idea that a given varicosity might have only a subset of kinases needed to phosphorylate all three active serine residues was invalidated by the experiment (Fig. $2 b$ ) in which all possible pairs of TH-P antibodies were tested. The vast majority of varicosities were positive for both. At present, we lack a testable hypothesis for the absence of TH-P in some varicosities.

The second main finding is that agents that either increase or decrease the firing rate of spiking retinal neurons modify $\mathrm{TH}$ phosphorylation. Because we did not directly measure the spiking rate, it might be argued that TTX and veratridine affected the neurons providing synaptic inputs to the DA neuron rather than, or in addition to, the DA neuron itself. Those inputs derive from two sorts of retinal neurons: bipolar cells, which are nonspiking (for review, see Dowling, 1987) and so not affected by TTXveratridine, and amacrine neurons of GABAergic and glycinergic subtypes (Gustincich et al., 1997). Had those amacrines been the targets of TTX, their inhibitory inputs to the DA neuron would have been reduced or eliminated, resulting in an increased phosphorylation of TH (Witkovsky et al., 2000) (compare with Fig. 6), the opposite result to what was obtained. Similarly, veratridine, if it acted on inputs to DA neurons, would be expected to increase inhibitory tone and reduce TH-P, whereas it was observed to increase it. Light also increases dopamine production and release (Kramer, 1971; this study) and TH-P (Whitkovsky et al., 2000), yet whether light effects these changes through alterations in spiking activity and/or other mechanisms has not yet been established.

\section{Relationship between electrical activity and TH-P formation in retinal DA neurons}

We noted an asymmetry between the actions of TTX and veratridine on TH-P formation, i.e., TTX selectively blocked it in the DA plexus, whereas veratridine increased it in all parts of the DA cell. To rationalize this difference, we suppose that $\mathrm{TH}-\mathrm{P}$ depends on depolarization. In the absence of spikes, DA cell bodies and dendrites still can be depolarized by excitatory synaptic inputs (or by the blockage of inhibitory inputs), whereas the synapse-free varicosities depend on spikes for depolarization. Veratridine increased TH-P throughout the DA neuron, presumably by increasing the spike rate and thereby increasing the level of depolarization throughout the neuron.

\section{Structural-functional organization of mammalian retinal dopaminergic neurons}

Dacey $(1988,1990)$ reported that cat and monkey retinal DA neurons had multiple axon-like processes that extended many millimeters from the perikaryon. The varicosities found along the axons and in the rings of the DA plexus are considered to be presynaptic sites on the basis of electron microscopic criteria (Dowling and Ehinger, 1978; Holmgren, 1982; Pourcho, 1982; Kolb et al., 1990). Dacey concluded that the rings were formed from axonal processes.

Synaptic input to the DA cells occurs onto the dendrites and perikarya, which together have a much more restricted spatial arborization than does the axonal arbor. Our findings that the 
varicosities contain both VMAT2 and a spiking Na channel supports the idea that the varicosities are sites of DA release and are capable of supporting regenerative action potentials. Given the absence of synaptic input to the varicosities (Pourcho, 1982; Kolb et al., 1990) and the great distance between DA axon terminals and their somata, a reasonable assumption is that the trains of action potentials passing down the axon regulate dopamine release. Piccolino et al. (1987) found that TTX blocked the dopamine-mediated effects of agents that increased release of endogenous dopamine, e.g., the $\mathrm{GABA}_{\mathrm{A}}$ antagonist bicuculline. Although the study by Piccolino et al. (1987) did not show directly that DA neurons produce spikes, subsequent studies have established that they do spike (Gustincich et al., 1997) and that TTX blocks dopamine release (Puopolo et al., 2001).

A corollary idea from the above considerations is that the axonal action potentials, by regulating the permeability of voltage-gated calcium channels, control the entry of calcium on which dopamine release is known to depend (Boatright et al., 1989; Tamura et al., 1995; Puopolo et al., 2001). Because a close coupling exists between catecholamine synthesis and release (Zigmond et al., 1989), it is plausible that TH-P also is regulated by calcium, an idea consistent with the known calcium dependence of some kinases. In that regard, Iuvone et al. (1982) showed that retinal tyrosine hydroxylase activity is increased by cAMP and protein kinase A (PKA), but a possible relationship between other kinases and retinal dopamine synthesis has not been reported. In the striatum, Haycock and Haycock (1991) found that TH-P at serine 40 was PKA dependent. In contrast, TH phosphorylation at serine 31 depends on MAP (mitogen-activated protein) kinase (Sutherland et al., 1993) and that at serine 19 depends on multiple kinases, including CaMKII (calcium/ calmodulin-dependent kinase) (for review, see Kumer and Vrana, 1996). It might be expected therefore that blockage of voltage-gated calcium channels would result in a reduction of retinal TH-P at serine 19 and perhaps also at serine 40, given that the most ubiquitous forms of adenylate cyclase in nervous tissue are calcium dependent. In preliminary tests, however, we found that intraocular injection of cobalt and/or cadmium, known blockers of voltage-gated calcium channels, did not reduce TH-P, as assessed by the three phosphospecific antibodies we used. Because our experiments did not provide a direct demonstration that calcium channels were completely blocked, however, a more definitive examination is called for. In addition, further studies are required to document the kinases, other than PKA, that subserve phosphorylation of retinal $\mathrm{TH}$.

\section{Release of dopamine from distal processes}

It is well established that rodent retinal dopaminergic neurons are of the inner plexiform subtype (Savy et al., 1995). That is, a few DA processes extend from the border of INL/IPL toward the photoreceptors, some reaching the photoreceptor layer, others ending in the INL. Such processes are similar to the DA axons in consisting of fine processes bearing occasional varicosities. We noted that VMAT2-IR was associated with some of the varicosities, including those in the middle of the INL (compare with Fig. $7 d$ ). Additional VMAT2-positive varicosities were found in the middle of the IPL. Our data suggest that dopamine may be released from the DA neurons at multiple sites, some quite far from their targets, e.g., the varicosities in the middle of the INL (Fig. $7 d)$. In fact, the whole question of the morphologically defined DA synapse needs reinvestigation in view of the findings that DA neurons also colocalize GABA (Wassle and Chun, 1988) and the report by Contini and Raviola (2003) that it is GABA receptors that cluster on the postsynaptic side opposite the DA-GABA presynaptic terminals. It therefore appears to be the case that DA diffuses over variable distances to its receptors, which may not be clustered but rather distributed more generally over the target neurons. It has been shown that the rings of the DA plexus surround mainly the AII amacrine cells (Tork and Stone, 1979), a subtype of amacrine neuron that is an integral component of the pathway processing retinal information under dim light conditions (for review, see Bloomfield and Dacheux, 2001). AII amacrine cells possess dopamine $\mathrm{D}_{1}$ receptors (Veruki and Wassle, 1996), and it has been shown that the permeability of the gap junctions linking neighboring AII amacrine cells is reduced by dopamine, acting through a $\mathrm{D}_{1}$ receptor (Hampson et al., 1992).

In conclusion, the DA amacrine cell has special properties associated with the role of its transmitter dopamine as a chemical messenger for light adaptation (Witkovsky, 2004). It is unique among known amacrine cells in having an endogenous spiking rhythm (Gustincich et al., 1997) but is similar in this respect to DA neurons of the nigrostriatal system in the CNS (Grace and Bunney, 1983). Its synaptic output appears to be governed by the spiking rate, which itself is an interaction between an endogenous rhythm and a mix of excitatory and inhibitory synaptic inputs. The retinal DA neuron evidently regulates its activity through its spiking frequency, which modulates the rates of both dopamine synthesis and dopamine release. By analogy with what has been shown for the nigrostriatal pathway (Floresco et al., 2003), bursts of spikes in retinal DA neurons might be expected to increase release beyond that evoked by steady firing; this is a subject for additional inquiry.

\section{References}

Akopian A, Witkovsky P (2001) Intracellular calcium reduces light-induced excitatory post-synaptic responses in salamander retinal ganglion cells. J Physiol (Lond) 532:43-53.

Bloomfield SA, Dacheux RF (2001) Rod vision: pathways and processing in the mammalian retina. Prog Retin Eye Res 20:351-384.

Boatright JH, Hoel MJ, Iuvone PM (1989) Stimulation of endogenous dopamine release and metabolism in amphibian retina by light- and $\mathrm{K}^{+}$ -evoked depolarization. Brain Res 482:164-168.

Boiko T, VanWart A, Caldwell HF, Levinson SR, Trimmer JS, Matthews G (2003) Functional specialization of the axon initial segment by isoformspecific sodium channel targeting. J Neurosci 23:2306-2313.

Contini M, Raviola E (2003) GABAergic synapses made by a retinal dopaminergic neuron. Proc Natl Acad Sci USA 100:1358-1363.

Dacey DM (1988) Dopamine-accumulating amacrine cells revealed by in vitro catecholamine-like fluorescence display a unique morphology. Science 240:1196-1198.

Dacey DM (1990) The dopaminergic amacrine cell. J Comp Neurol 301:461-489.

Dowling JE (1987) The retina. An approachable part of the brain, Chap 4. Cambridge, MA: Belknap.

Dowling JE, Ehinger B (1978) Synaptic organization of the dopaminergic neurons in the rabbit retina. J Comp Neurol 180:203-220.

Erickson JD, Schafer MK-H, Bonner TI, Eiden LE, Weihe E (1996) Distinct pharmacological properties and distribution in neurons and endocrine cells of two isoforms of the human vesicular monoamine transporter. Proc Natl Acad Sci USA 93:5166-5171.

Feigenspan A, Gustincich S, Raviola E (2000) Pharmacology of GABA $\mathrm{A}$ receptors of retinal dopaminergic neurons. J Neurophysiol 84:1697-1707.

Floresco SB, West AR, Ash B, Moore H, Grace AA (2003) Afferent modulation of dopamine neuron firing differentially regulates tonic and phasic dopamine transmission. Nat Neurosci 6:968-973.

Grace AA, Bunney BS (1983) Intracellular and extracellular electrophysiology of nigral dopaminergic neurons. 1. Identification and characterization. Neuroscience 10:301-315.

Gustincich S, Feigenspan S, Wu DK, Koopman LJ, Raviola E (1997) Control of dopamine release in the retina: a transgenic approach to neural networks. Neuron 18:723-736. 
Hampson ECGM, Vaney DI, Weiler R (1992) Dopaminergic modulation of gap junction permeability between amacrine cells in mammalian retina. J Neurosci 12:4911-4922.

Haycock JW, Haycock DA (1991) Tyrosine hydroxylase in rat brain dopaminergic nerve terminals. Multiple-site phosphorylation in vivo and in synaptosomes. J Biol Chem 266:5650-5657.

Haycock JW, Becker L, Ang L, Furukawa Y, Hornykiewicz O, Kish SJ (2003) Marked disparity between age-related changes in dopamine and other presynaptic dopaminergic markers in striatum. J Neurochem 87:574-585.

Hille B (1992) Ionic channels of excitable membranes, Chap 17. Sunderland, MA: Sinauer.

Hoffman BJ, Hansson SR, Mezey E, Palkovits M (1998) Localization and dynamic regulation of biogenic amine transporters in the mammalian central nervous system. Front Neuroendocrinol 19:187-231.

Holmgren I (1982) Synaptic organization of the dopaminergic neurons in the retina of the cynomolgus monkey. Invest Ophthalmol Vis Sci 22:8-24.

Iuvone PM, Rauch AL, Marshburn PB, Glass DB, Neff NH (1982) Activation of retinal tyrosine hydroxylase in vitro by cyclic AMP-dependent protein kinase: characterization and comparison to activation in vivo by photic stimulation. J Neurochem 39:1632-1640.

Kolb H, Cuenca N, Wang HH, Dekorver L (1990) The synaptic organization of the dopaminergic amacrine cell in the cat retina. J Neurocytol 19:343-366.

Kramer SG (1971) Dopamine: a retinal neurotransmitter. I. Retinal uptake, storage, and light-stimulated release of $\mathrm{H} 3$-dopamine in vivo. Invest Ophthalmol 10:438-452.

Kumer SC, Vrana KE (1996) Intricate regulation of tyrosine hydroxylase activity and gene expression. J Neurochem 74:443-462.

Mariani AP, Hokoc JN (1988) Two types of tyrosine hydroxylaseimmunoreactive amacrine cells in the rhesus monkey retina. J Comp Neurol 276:81-91.

Nguyen-Legros J, Botteri-Versaux C, Vernier P (1999) Dopamine receptor localization in the mammalian retina. Mol Neurobiol 19:181-204.

Nir I, Haque R, Iuvone PM (2000a) Diurnal metabolism of dopamine in the mouse retina. Brain Res 870:118-125.

Nir I, Haque R, Iuvone PM (2000b) Diurnal metabolism of dopamine in dystrophic retinas of homozygous and heterozygous retinal degeneration slow (rds) mice. Brain Res 884:13-22.

Piccolino M, Witkovsky P, Trimarchi C (1987) Dopaminergic mechanisms underlying the reduction of electrical coupling between horizontal cells of the turtle retina induced by $\mathrm{d}$-amphetamine, bicuculline, and veratridine. J Neurosci 7:2273-2285.

Pourcho RG (1982) Dopaminergic amacrine cells in the cat retina. Brain Res 252:101-109.

Puopolo M, Hochstetler SE, Gustincich S, Wightman RM, Raviola E (2001) Extrasynaptic release of dopamine in a retinal neuron: activity dependence and transmitter modulation. Neuron 30:211-225.

Salvatore MF, Garcia-Espana A, Goldstein M, Deutch AY, Haycock JW (2000) Stoichiometry of tyrosine hydroxylase phosphorylation in nigrostriatal and mesolimbic systems in vivo: effects of haloperidol and related compounds. J Neurochem 75:225-232.

Savy C, Moussafi F, Durand J, Yelnik J, Simon A, Nguyen-Legros J (1995) Distribution and spatial geometry of dopamine interplexiform cells in the retina. II. External arborizations in the adult rat and monkey. J Comp Neurol 355:392-404.

Sutherland C, Alterio J, Campbell DG, LeBourdelles B, Mallet J, Haavik J, Cohen P (1993) Phosphorylation and activation of human tyrosine hydroxylase in vitro by mitogen-activated protein (MAP) kinase and MAPkinase-activated kinases 1 and 2. Eur J Biochem 217:715-722.

Tamura N, Yokotani K, Okuma Y, Okada M, Ueno H, Osumi Y (1995) Properties of the voltage-gated calcium channels mediating dopamine and acetylcholine release from the isolated rat retina. Brain Res 767:363-370.

Tork I, Stone J (1979) Morphology of catecholamine-containing amacrine cells in the cat's retina. Brain Res 169:261-273.

Veruki M, Wassle H (1996) Immunohistochemical localization of dopamine D1 receptors in rat retina. Eur J Neurosci 8:2286-2297.

Wassle H, Chun MH (1988) Dopaminergic and indoleamine-accumulating amacrine cells express GABA-like immunoreactivity in the cat retina. J Neurosci 8:3383-3394.

Witkovsky P (2004) Dopamine and retinal function. Doc Ophthalmol 108:17-39.

Witkovsky P, Gabriel R, Haycock J, Meller E (2000) Influence of light and neural circuitry on tyrosine hydroxylase phosphorylation in the rat retina. J Chem Neuroanat 19:105-116.

Zigmond RE, Schwarzschild MA, Rittenhouse AR (1989) Acute regulation of tyrosine hydroxylase by nerve activity and by neurotransmitters via phosphorylation. Annu Rev Neurosci 12:415-461. 